\title{
Commentary on the article "Pacemaker Lead as an latrogenic Cause of Right Heart Failure" and Review of Pacing-Induced Heart Failure Manuel Toscano*
}

Medical Resident, Internal Medicine Department, Hospital de Cascais, Lisboa, Portuga

\section{Article Info}

\section{Article Notes}

Received: December 20, 2019

Accepted: January 9, 2020

*Correspondence:

Dr. Manuel Toscano, Rua D. Francisco de Avilez, $n^{0}$ 26, $2^{\circ}$ esq, 2750-349 Cascais, Portugal; Telephone No: +351918221728; Email: manuel2toscano@gmail.com.

c 2020 Toscano M. This article is distributed under the terms of the Creative Commons Attribution 4.0 International License.
Patients presenting with right-sided heart failure represent a clinical challenge, with numerous potential etiologies and limited treatment options. Although technical advances in healthcare have improved patient survival and quality of life, they are not innocuous and even routine procedures may cause potentially irreversible complications. Right ventricular pacing (RVP), for example, has been common practice for several decades, but its benefits and safety have been frequently called into question. This matter has become more pressing over the past decades as the implantation of cardiac implantable electronic devices has grown due to expanding indications and increased life expectancy.

This article aims to review tricuspid regurgitation (TR) related to permanent pacemaker (PPM)/implantable cardioverter-defibrillator (ICD) leads and pacing-induced cardiomyopathy (PICM), which are clinically important but under-recognized iatrogenic etiologies of heart failure.

\section{Prevalence}

The overall prevalence of TR in patients with PPM has been estimated between $25 \%$ and $29 \%$ and numerous studies have found worsening of pre-existing TR by 1-2 grades in up to $25 \%$ of patients with newly implanted devices ${ }^{1,2}$. ICD appears to cause TR more frequently than PPM, mainly due to differences in lead size. Of note, new-onset or worsening of existing TR may occur up to 7 years following device implantation, which highlights the need for a high index of suspicion in patients who have had devices for several years ${ }^{3}$. Early detection of this complication is of the utmost importance as prognosis worsens with right ventricular failure and 10 -year survival is 30 to $50 \%{ }^{4}$.

The impact of PICM may also be greater than previously anticipated. Khurshid et al. reported a prevalence of approximately $20 \%$ of patients, with RVP time as little as $20 \%$, findings that were confirmed in other trials ${ }^{5-7}$.

\section{Pathophysiology}

Although current literature regarding lead-related TR following PPM or ICD implantation is limited, surgical and autopsy series suggest that lead impingement and lead adherence are the most common causes and that more severe TR is seen when the leads impinge on the tips of the tricuspid valve leaflets ${ }^{8}$.

On the other hand, worsening TR has also been attributed to the mode and percentage of RVP, which causes valve dysfunction 


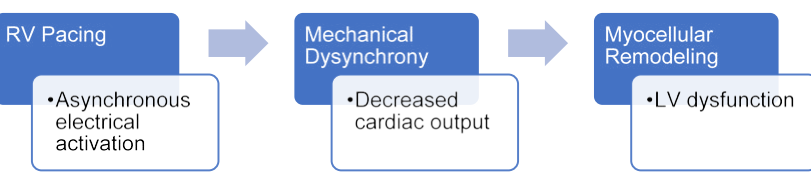

Figure 1. Pathophysiology of Pacing-Induced Cardiomyopathy

either through delayed right ventricular (RV) activation and ventricular desynchrony or changes in RV geometry, leading to PICM (Figure 1).

Paced QRS duration appears to be a major determinant in the development of PICM while patients' age and male gender have also been identified as possible risk factors 9 . Despite this, few and controversial data exist regarding the long-term effect of chronic RVP on the left ventricular systolic function of patients with preserved left ventricular ejection fraction (LVEF) at the time of PPM implantation. More importantly, it is not clear if, how and when the negative left ventricular (LV) remodeling will lead to PICM and heart failure symptoms since, as shown by Khurshid et al., only half of the patients with echocardiographic diagnosis of PICM will present clinical evidence of overt heart failure symptoms ${ }^{5}$. In the multicenter PREVENT-HF trial, chronic RVP had no effect on the 12-month followup $\operatorname{LVEF}^{10}$. The same year, the PACE trial, also involving patients with preserved LVEF, reported the superiority of bi-ventricular pacing to RVP in the prevention of LV adverse remodeling and deterioration of systolic function at 2-year follow-up ${ }^{7}$. Lu et al. recently observed no correlation between baseline LVEF and mortality or heart failure hospitalization in patients with either bi-ventricular or $\mathrm{RVP}^{11}$. In contrast, studies such as the DAVID trial had shown that the deleterious effects of RVP are particularly evident in patients with severely reduced LVEF $(<40 \%)$ and that mortality and heart failure was higher with dual-chamber ICD than with single-chamber ICD ${ }^{12}$. Furthermore, the MOST trial also showed that reduced LVEF predicts sudden cardiac death and heart failure occurrence in patients with sinus node disease implanted with RV apical PPM ${ }^{13}$. These two studies and trials such as SAVE-PACe formed the basis for the opinion that RVP should be avoided by all means ${ }^{14}$.

\section{Treatment}

Current valvular guidelines recommend concomitant tricuspid valve repair in the setting of mild to severe TR when there is evidence of tricuspid annular dilation or prior evidence of right-sided heart failure ${ }^{15}$. Minimally invasive surgery through right mini-thoracotomy has gained popularity and constitutes a safe alternative to conventional sternotomy, allowing direct and unimpaired anatomical visualization of both the mitral and tricuspid valves, as well as reduced surgical trauma, blood loss and pain, shorter hospital length of stay and a more rapid return to functional activity. This approach has also been used successfully in patients requiring isolated tricuspid valve surgery, with mortality rates of $8.2-9.5 \%{ }^{16,17}$. If possible, removal of the trans-tricuspid lead and replacement with an epicardial lead at the time of tricuspid valve surgery may reduce late repair failure ${ }^{18}$. However, it is worth mentioning that lead extraction is not routinely recommended in transtricuspid lead-induced severe TR, due to procedural risks, especially if the leads are adherent to the valve leaflets.

Regarding PICM, a meta-analysis of seven randomized trials on the prevention of RVP failed to show any significant impact on clinical endpoints ${ }^{19}$. Hence, despite clear evidence of the deleterious effects of RVP, namely ventricular desynchrony, deterioration of hemodynamic measurements and histopathological alterations, there appears to be no benefit in preventing RVP.

\section{Conclusion}

In sum, despite several years of research and multiple studies involving thousands of patients, the optimal pacing strategy has yet to be defined. New-onset or worsening TR remains a frequent but often unpredictable complication of RV lead placement. On the other hand, there is still controversy surrounding PICM and its prevention. Identifying key risk factors for LVEF reduction and development of PICM may someday help guide decisions to implant a dual-chamber device, instead of a simple RV apical PPM, in select patients even with LVEF $>35 \%$ and QRS $<120 \mathrm{~ms}$.

\section{Conflict of Interest}

I confirm that this text has not been submitted for publication in whole or in part in another journal.

I guarantee there is no intentional plagiarism in this article and that all the references used were cited.

There were no conflicts of interest or sources of financing in this work.

\section{References}

1. Paniagua D, Aldrich HR, Lieberman EH, et al. Increased prevalence of significant tricuspid regurgitation in patients with transvenous pacemakers leads. Am J Cardiol. 1998; 82: 1130-1132.

2. Kim JB, Spevack DM, Tunick PA, et al. The effect of transvenous pacemaker and implantable cardioverter defibrillator lead placement on tricuspid valve function: an observational study. J Am Soc Echocardiogr. 2008; 21: 284-287.

3. Grupper A, Killu AM, Friedman PA, et al. Effect of tricuspid valve regurgitation on outcome in patients with cardiac resynchronization therapy. Am J Cardiol. 2015; 115(6): 783-789.

4. McCarthy PM, Bhudia SK, Rajeswaran J, et al. Tricuspid valverepair: durability and risk factors for failure. J Thorac Cardio-vasc Surg. 2004.

5. Khurshid S, Liang JJ, Owens A, et al. Longer paced QRS duration is associated with increased prevalence of right ventricular pacinginduced cardiomyopathy. J Cardiovasc Electrophysiol. 2016; 27: 1174-9.

6. Kim JH, Kang KW, Chin JY, et al. Major determinant of the occurrence 
of pacing-induced cardiomyopathy in complete atrioventricular block: a multicentre, retrospective analysis over a 15-year period in South Korea. BMJ Open. 2018; 8: e019048.

7. Chan JYS, Fang F, Zhang Q, et al. Biventricular pacing is superior to right ventricular pacing in bradycardia patients with preserved systolic function: 2-year results of the PACE trial. Eur Heart J. 2011; 32: $2533-40$

8. Al-Bawardy R, Krishnaswamy A, Bhargava M, et al. Tricuspid Regurgitation in Patients With Pacemakers and Implantable Cardiac Defibrillators: A Comprehensive Review. Clin Cardiol. 2013; 36: 249 254

9. Merchant FM, Mittal S. Pacing-induced cardiomyopathy. Card Electrophysiol Clin. 2018; 10: 437-45.

10. Stockburger M, Gomez-Doblas JJ, Lamas G, et al. Preventing ventricular dysfunction in pacemaker patients without advanced heart failure results from a multicentre international randomized trial (PREVENTHF). Eur J Heart Fail. 2011; 13: 633-41.

11. Lu D, Zhang $\mathrm{H}$, Chen $\mathrm{C}$, et al. Clinical outcomes with biventricular versus right ventricular pacing in patients with atrioventricular conduction defects. Heart Fail Rev. 2018; 23: 897-906.

12. Wilkoff BL, Cook JR, Epstein AE, et al. Dual-chamber pacing or ventricular backup pacing in patients with an implantable defibrillator: the Dual Chamber and VVI Implantable Defibrillator (DAVID) Trial. JAMA. 2002; 288: 3115-23.
13. Sweeney MO, Hellkamp AS, Ellenbogen KA, et al. Adverse effect of ventricular pacing on heart failure and atrial fibrillation among patients with normal baseline QRS duration in a clinical trial of pacemaker therapy for sinus node dysfunction. Circulation. 2003, 107(23): 2932-7.

14. Sweeney MO, Bank AJ, Nsah E, et al. Minimizing ventricular pacing to reduce atrial fibrillation in sinus-node disease. N Engl J Med. 2007; 357: 1000-8.

15. Baumgartner H, Falk V, Bax JJ, et al. ESC Scientific Document Group 2017 ESC/EACTS Guidelines for the management of valvular heart disease. Eur Heart J. 2017; 38: 2739-91.

16. Vassileva CM, Shabosky J, Boley T, et al. Tricuspid valve surgery: the past 10 years from the Nationwide Inpatient Sample (NIS) database. ] Thorac Cardiovasc Surg. 2012; 143: 1043-9.

17. Kilic A, Saha-Chaudhuri P, Rankin JS, et al. The Annals of Thoracic Surgery, Trends and Outcomes of Tricuspid Valve Surgery in North America: An Analysis of More Than 50,000 Patients From The Society of Thoracic Surgeons Database. 2013; 5: 1546-52.

18. Singh SK, Tang GH, Maganti MD, et al. Midterm outcomes of tricuspid valve repair versus replacement for organic tricuspid disease. Ann Thorac Surg. 2006; 82: 1735-1741.

19. Shurrab M, Healey JS, Haj-Yahia S, et al. Reduction in unnecessary ventricular pacing fails to affect hard clinical outcomes in patients with preserved LV function: a meta-analysis. Europace. 2016. 